\title{
Mapeamento Sistemático sobre Interfaces Tangíveis para apoiar o ensino do Braille
}

\author{
Franciele Beal $^{1,2}$, Laura Sánchez García ${ }^{1}$ \\ ${ }^{1}$ Programa de Pós-graduação em Informática - Universidade Federal do Paraná (UFPR) \\ 80.050-540 - Curitiba - PR - Brasil. \\ ${ }^{2}$ Universidade Tecnológica Federal do Paraná (UTFPR), Câmpus Dois Vizinhos (DV), \\ Coordenadoria de Engenharia de Software (COENS), PR - Brasil. \\ fbealdutfpr.edu.br, lauradinf.ufpr.br
}

\begin{abstract}
A phenomenon called "desbrailização"has generated negative consequences for the education of the blind. It's the replacement of Braille with audio technologies. Braille is indispensable for the education of the blind. Thus, in this work a systematic mapping of studies published in the last 10 years in the area of Tangible User Interface as an educational resource was carried out for teaching Braille.The results reveal a small amount of work in the area, suggesting a research opportunity to be explored.
\end{abstract}

Resumo. Um fenômeno chamado de "desbrailização"tem gerado consequências negativas na educação dos cegos. Trata-se da substituição do Braille por tecnologias de áudio. O Braille é indispensável para a educação do cego. Desta forma, este trabalho realizou um mapeamento sistemático de estudos publicados nos últimos 10 anos na área de Interfaces Tangíveis como recurso educacional para ensino do Braille. Os resultados revelam uma pequena quantidade de trabalhos na área, sugerindo uma oportunidade de pesquisa a ser explorada.

\section{Introdução}

O Braille [American Foundation for the Blind 2019] é o sistema de escrita e leitura em relevo utilizado pelos cegos que é interpretado pelo tato. Letras e símbolos são representados em um espaço retangular chamado de cela Braille que é forma por 6 pontos distribuídos em 3 linhas e 2 colunas.

Em complemento ao Braille, existem as tecnologias de áudio que tem como objetivo possibilitar o acesso às informações e ao conhecimento que estão disponíveis em meio digital por meio de softwares que fazem a leitura do que está na tela do computador. Com elas ficou mais fácil ouvir que ler em Braille e isso está fazendo com que o Braille seja subtituído gerando a "desbrailização"[Davanzo Batista 2018]. A "desbrailização"coloca em risco a educação dos cegos porque o texto áudio não ensina a ler e a escrever. Ele não possibilita reconhecer a ortografia das palavras e a estrutura de um texto [Martinez et al. 2018]. Desta forma, as tecnologias de áudio não são suficientes para a alfabetização.

O Braille é indispensável para a educação dos cegos. Segundo a OMS, pessoas cegas têm mais chances de vivenciar taxas mais altas de pobreza e levar uma vida marcada por desigualdades. De acordo com a Convenção Internacional sobre os Direitos das 
VIII Congresso Brasileiro de Informática na Educação (CBIE 2019)

Anais do XXX Simpósio Brasileiro de Informática na Educação (SBIE 2019)

Pessoas com Deficiência, o Braille é essencial porque é um meio de comunicação, liberdade de expressão e opinião, que possibilita o acesso à informação e a inclusão social [Nações Unidas do Brasil 2019]. A pessoa cega que é fluente em Braille e faz o uso dele tem mais chances de obter sucesso, independência, maior probabilidade de conseguir emprego, renda maior e satisfação no trabalho [Stanfa e Johnson 2017]. Além disso, o Braille melhora a autoestima e o bem estar dessas pessoas cegas[Silverman e Bell 2018].

Considerando a importância do Braille para o cego e o problema da "desbrailização", acredita-se que o resgate do Braille em atividades de alfabetização e principalmente de letramento ${ }^{1}$, pode ajudar a desenvolver e trazer melhorias na leitura e na escrita Braille.

Além disso, a utilização de tecnologia com interação tangível pode estimular e favorecer o aprendizado do Braille. Uma Interface Tangível (TUI - Tangible User Interface) tem como objetivo dar forma física para informações digitais para que possam ser manipuladas por meio de objetos físicos [Ishii 2008] o que pode torná-la adequada aos cegos. Ademais, segundo [Moreira e Baranauskas 2015], as TUIs tem se destacado como recurso auxiliar em ambientes educacionais inclusivos.

Desta forma, a pesquisa aqui relatada investigou trabalhos que utilizam TUI, para responder as seguintes questões de pesquisa: Quais TUIs existem para atividades de alfabetização ou letramento Braille? Quais são suas características e limitações para apoiar atividades de letramento Braille? Para a investigação proposta, optou-se por um Mapeamento Sistemático (MS) para categorizar e sintetizar informações existentes sobre o uso de TUI para o ensino do Braille e revelar lacunas desse espaço de pesquisa. $O$ MS incluiu estudos publicados em bases digitais nacionais e internacionais da área de Informática na Educação entre os anos 2010 e 2019. A busca foi realizada de forma automática em quatro bases que retornaram 382 artigos. Após aplicação de critérios de inclusão e exclusão foram selecionados 10 artigos que são apresentados neste trabalho que está organizado da seguinte forma: na Seção 2 é descrita a metodologia de pesquisa. A Seção 3 apresenta os resultados do mapeamento e a discussão. Na Seção 4 a conclusão e trabalhos futuros.

\section{Metodologia de Pesquisa}

Este trabalho utilizou como base as diretrizes apresentadas por [Kitchenham e Charters 2007] e seu objetivo está baseado no paradigma Goal-QuestionMetric, ou GQM [Basili e Rombach 1988]: analisar ambientes computacionais baseados em TUI com o propósito de identificar e caracterizar em relação a tecnologias e recomendações para a construção de TUI para o ensino do Braille do ponto de vista de pesquisadores da área de Interação Humano Computador (IHC) e designers no contexto de fontes primárias (com foco em Informática na Educação, IHC e TUI) disponíveis em bases digitais.

\footnotetext{
${ }^{1}$ Este trabalho segue as definições de alfabetização e letramento propostas por [Soares 1999]. A alfabetização compreende atividades para a aquisição do sistema Braille por meio da codificação e decodificação da escrita. Já o letramento é o desenvolvimento do uso competente da leitura e da escrita Braille em práticas sociais [Soares 1999]. Outros autores também reforçam a diferença do letramento para a alfabetização [Foucambert 2008] e [Freire 1987].
} 
VIII Congresso Brasileiro de Informática na Educação (CBIE 2019)

Anais do XXX Simpósio Brasileiro de Informática na Educação (SBIE 2019)

\subsection{Questões de Pesquisa}

A partir das questões de pesquisa principais foram definidas outras cinco questões específicas:

- QP1: Qual a tecnologia (RFID, visão computacional, microcontroladores, entre outras) utilizada na implementação da TUI?

- QP2: Como as crianças interagem com o ambiente e quais tipos de feedback são dados à elas?

- QP3: Como esses trabalhos foram testados e por quem?

- QP4: Possui limitações em relação ao apoio às atividades de letramento?

- QP5: Os trabalhos apresentam recomendações ou requisitos para o projeto de TUIs para cegos?

\subsection{Definição da String de Busca e das Bases}

Para a definição das palavras-chave e o agrupamento dos termos para formar a string de busca foi utilizada a metodologia PICOC (População, Intervenção, Comparação, Resultado e Contexto) proposto por [Kitchenham e Charters 2007]: (a) População: "blind children", "blind people", blind, "visually impaired", "visual impairments", blindness, braille; (b) Intervenção: "tangible interaction", "tangible user interface”, "tangible interface", "tangible technology", TUI; (c) Resultados: education, "educational tecnology”, teaching, learning, literacy. Onde, População se refere aos usuários para os quais as TUIs são projetadas; Intervenção se refere ao que se quer encontrar; e, Resultados corresponde o que ser quer atender, ou melhorar. O termo Comparação não se aplica, pois, o objetivo é categorizar as TUI, não compará-las. O termo Contexto não se aplica devido o fato de não haver comparação.

Para obter os estudos primários relevantes para essa pesquisa, foram utilizadas as seguintes bibliotecas digitais: IEEExplore ${ }^{2}$, Scopus ${ }^{3}$, Web of Science $^{4}$ e o Portal de Publicações da CEIE 5 .

Os idiomas escolhidos foram o Inglês e o Português. O Inglês foi escolhido por ser o idioma mais utilizado em conferências e periódicos da área. Já o Português, foi escolhido porque este trabalho está sendo desenvolvido no Brasil e se quer investigar estudos em nível nacional. Desta forma, para a base CEIE foi utilizada tanto string de busca em Português, quanto em Inglês. As palavras no plural foram adicionadas explicitamente na string de busca. Para a busca na base CEIE foi construída uma string auxiliar juntando os termos em inglês e português. As strings de buscas utilizadas são apresentadas (Tabela $1)$ :

Na base CEIE foi utilizado o asterísco * para tratar palavras no plural e também para reduzir o tamanho da string porque o campo de busca no site tem tamanho limitado.

\footnotetext{
${ }^{2}$ IEEExplorer - http://ieeexplore.ieee.org/Xplore/home.jsp

${ }^{3}$ Scopus - https://www.scopus.com/

${ }^{4} \mathrm{https}$ ://clarivate.com/products/web-of-science/

${ }^{5} \mathrm{CEIE}$ - http://br-ie.org/pub/index.php/index/search Armazena trabalhos de CBIE (Congresso Brasileiro de Informática na Educação), RBIE (Revista Brasileira de Informática na Educação), SBIE (Simpósio Brasileiro de Informática na Educação) e WIE (Workshop de Informática na Escola)
} 
VIII Congresso Brasileiro de Informática na Educação (CBIE 2019)

Anais do XXX Simpósio Brasileiro de Informática na Educação (SBIE 2019)

Tabela 1. Strings de busca utilizadas nas bases

\begin{tabular}{|c|c|}
\hline Bases & String \\
\hline $\begin{array}{l}\text { IEEExplorer, } \\
\text { Scopus e Web } \\
\text { of Science }\end{array}$ & $\begin{array}{l}\text { ("tangible interaction"OR "tangible user interface"OR "tangible } \\
\text { interface"OR "tangible technology"OR "tangibles users interfaces" } \\
\text { OR "tangibles interfaces"OR "tangibles technologies"OR TUI) } \\
\text { AND (education OR "educational tecnology"OR "educational } \\
\text { technologies"OR teaching OR learning OR literacy) AND } \\
\text { ("blind children"OR "blind child"OR "blind people"OR blind } \\
\text { OR "visually impaired"OR "visual impairments"OR blindness } \\
\text { OR braille) }\end{array}$ \\
\hline CEIE & $\begin{array}{l}\text { (tangibl* OR *tangi* OR TUI) AND }(* \text { educa* OR teaching OR } \\
\text { ensino OR learning OR aprendizagem OR literacy OR alfabetização } \\
\text { OR letramento) AND (blind* OR *cega* OR cego* OR cegueira } \\
\text { OR visua*impair* OR defici*visua* OR braille) }\end{array}$ \\
\hline
\end{tabular}

\subsection{Seleção dos Estudos}

Os estudos retornados com a aplicação das strings de buscas em cada uma das bases foram analisados um a um, considerando o título e o abstract. Para garantir uma seleção imparcial, foram definidos critérios de inclusão e de exclusão de estudos. Critérios de inclusão:

- Artigos que apresentam TUI em sala de aula em atividades de alfabetização ou letramento Braille;

- Artigos que apresentam requisitos para a construção de TUI para crianças cegas.

Critérios de exclusão:

- Relatórios técnicos, apresentações, índices,notas e comentários;

- Artigos com mais de 10 anos;

- Artigos duplicados;

- Artigos que não estão em português, ou inglês;

- Versões mais antigas do artigo;

- Artigos sem acesso (link indisponível, não disponibilizado pelas máquinas de buscas, etc.);

- Artigos que não estão relacionados com o uso de TUI como recurso educacional para cegos ou que não contribuem para orientar o processo de construção.

A pesquisa foi realizada no período do dia 02/05/2019 ao dia 07/05/2019. Foram retornados automaticamente 382 artigos. Na sequência foi realizada a leitura dos títulos e dos resumos e foram aplicados os critérios de inclusão e exclusão. Foi gerada uma lista com 47 estudos selecionados. Na sequência, foi realizada a leitura integral dos artigos selecionados e os critérios de inclusão e exclusão foram aplicados novamente. Durante o processo de leitura integral 37 estudos foram rejeitados e enquadrados em critérios de exclusão, sendo 10 estudos aceitos. A próxima seção apresenta os dados e informações extraídos desses estudos.

\section{Resultados do Mapeamento}

De acordo com os dados extraídos dos estudos, eles foram publicados entre os anos 2010 e 2018 (Figura 1). Como pode-se perceber foram publicados estudos nos últimos 3 anos o 
VIII Congresso Brasileiro de Informática na Educação (CBIE 2019)

Anais do XXX Simpósio Brasileiro de Informática na Educação (SBIE 2019)

que indica que as tecnologias tangíveis vem sendo estudadas em ambientes educacionais inclusivos e que existem pesquisas recentes. Essa informação serve de motivação para seguir na linha das TUI.

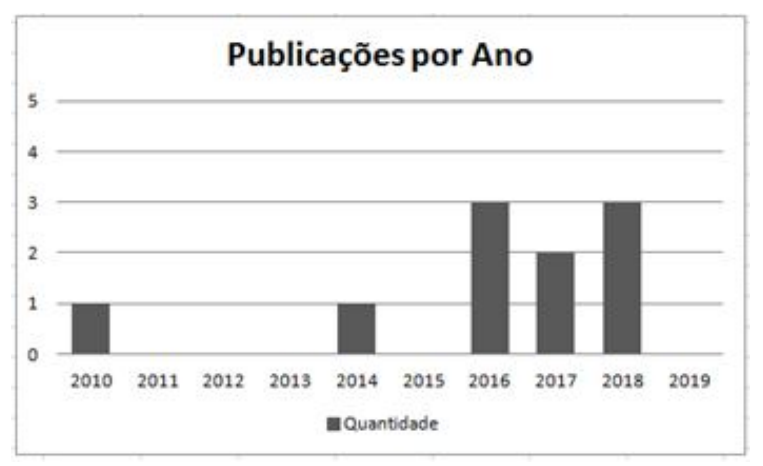

Figura 1. Quantidade de publicações por ano

Em relação à localização geográfica das publicações, 2 estudos são de pesquisadores da Arábia Saudita, outros 2 estudos são do Brasil e os demais são de pesquisadores da Alemanha, Espanha, Finlândia, França, Paquistão e Suécia.

\subsection{Respondendo às Perguntas de Pesquisa}

Este MS teve como objetivo responder as questões de pesquisa apresentadas na seção 2. Desta forma, os dados foram extraídos com base nessas questões. Para tal os trabalhos foram divididos em 2 grupos. O primeiro grupo é formado por 5 trabalhos ([Jafri 2014], [Sánchez García et al. 2016], [Forcelini et al. 2018], [Lozano et al. 2018] e [Maher et al. 2018]) que apresentam soluções de TUI para alfabetização e letramento Braille. Esse grupo é usado para responder todas as questões de pesquisa. Os demais trabalhos formam o grupo 2 ([McGookin et al. 2010], [Brulé et al. 2016], [Rühmann et al. 2016], [Ávila-Soto et al. 2017] e [Jafri et al. 2017]). Esses trabalhos são sobre TUI para cegos, porém, são voltados ao ensino de outros conceitos como geometria, funções matemáticas e geografia. Apesar de não serem voltados ao ensino do Braille eles foram selecionados porque apresentam informações relevantes para responder a QP5.

A Tabela 2 apresenta a descrição dos trabalhos do grupo 1.

A Tabela 3 apresenta a resposta da QP1 sobre as tecnologias utilizadas, ou sugeridas, na construção das TUIs.

Sobre como as crianças interagem com esses ambientes e quais tipos de feedback são fornecido à elas (resposta da QP2), os trabalhos [Jafri 2014], [Sánchez García et al. 2016], [Forcelini et al. 2018] e [Maher et al. 2018] fornecem interação por toque e áudio. [Lozano et al. 2018] fornece interação por toque, fala e áudio. A interação por meio do toque diz respeito a pegar o tangível, sentir sua forma e/ou informações táteis e posicionar na superfície. No caso do trabalho de [Maher et al. 2018], a interação por toque ocorre por meio de botões presentes na cela Braille eletrônica que são pressionados para formar o caracter Braille enviado ao computador. A interação por fala se refere à respostas faladas. $\mathrm{O}$ áudio foi utilizado para orientar os usuários durante a utilização das TUIs e dar feedback de acordo com as suas ações.

\footnotetext{
${ }^{6} \mathrm{OPENCV}$ - https://sourceforge.net/projects/opencvlibrary/
} 
VIII Congresso Brasileiro de Informática na Educação (CBIE 2019)

Anais do XXX Simpósio Brasileiro de Informática na Educação (SBIE 2019)

Tabela 2. Trabalhos sobre TUI para alfabetização e letramento Braille

\begin{tabular}{|l|l|l|}
\hline Trabalho & Descrição & Escopo \\
\hline Jafri (2014) & $\begin{array}{l}\text { Aplicativo baseado em interface tangível para ensinar } \\
\text { o reconhecimento de letras em Braille para crianças } \\
\text { cegas. }\end{array}$ & Alfabetização \\
\hline $\begin{array}{l}\text { Sánchez García } \\
\text { et al. (2016) }\end{array}$ & $\begin{array}{l}\text { Uma plataforma de interação tangível como apoio à } \\
\text { atividades de construção de textos em Braille para } \\
\text { crianças cegas. }\end{array}$ & Letramento \\
\hline $\begin{array}{l}\text { Forcelini et al. } \\
(2018)\end{array}$ & $\begin{array}{l}\text { Arquitetura de ambiente interativo tangível de baixo } \\
\text { custo para apoiar atividades de construção de textos } \\
\text { em Braille para combater a "desbrailização”. }\end{array}$ & Letramento \\
\hline $\begin{array}{l}\text { Lozano et al. } \\
\text { (2018) }\end{array}$ & $\begin{array}{l}\text { TUI para facilitar a aprendizagem de números em } \\
\text { Braille, texturas, formas e geometria para crianças } \\
\text { com deficiência visual. }\end{array}$ & Alfabetização \\
\hline $\begin{array}{l}\text { Maher et al. } \\
\text { (2018) }\end{array}$ & $\begin{array}{l}\text { Um framework de baixo custo baseado em TUI } \\
\text { para apoiar a escrita Braille para crianças com } \\
\text { deficiência visual. }\end{array}$ & Letramento \\
\hline
\end{tabular}

Outra informação investigada nesse estudo foi saber com quem foram realizados os testes nos ambientes (resposta da QP3). [Jafri 2014] realizou consultoria com professores de Braille e não apresenta resultados de testes em protótipo. [Sánchez García et al. 2016] descreveram entrevista semiestruturada e teste em protótipo com professor cego. [Forcelini et al. 2018] realizaram teste em protótipo com professores e crianças cegas. [Lozano et al. 2018] não apresenta resultados de testes na funcionalidade números em Braille. Em [Maher et al. 2018] foi relatado teste em protótipo com crianças cegas.

Os trabalhos apresentados na Tabela 2 também foram analisados para identificar limitações em relação ao apoio às atividades de letramento Braille (QP4). Os trabalhos de [Jafri 2014] e [Lozano et al. 2018] são voltados ao apoio da alfabetização. [Sánchez García et al. 2016] e [Forcelini et al. 2018] são propostas de soluções para apoiar vários tipos de atividade de letramento que envolvem basicamente a construção e a leitura crítica de textos significativos em Braille, sendo limitado como apoio a atividades que envolvam construções mais complexas como mapas conceituais. [Maher et al. 2018] é voltado ao ensino da escrita Braille. A leitura do que é escrito é feita por sintetizador de voz com a possibilidade de realizar a impressão em Braille para fazer a leitura tátil e apresenta a mesma limitação de [Sánchez García et al. 2016] e [Forcelini et al. 2018] em relação a atividades de letramento e de representação do conhecimento de maneira geral com construções em Braille mais complexas.

Para responder a QP5 foram utilizados os trabalhos dos Grupo 1 e Grupo 2. Os trabalhos revisados no MS trouxeram requisitos e recomendações úteis para orientar o design e a implementação de TUI para cegos:

- Os objetos tangíveis devem ser estáveis e com encaixes fortes e firmes para não desmontar facilmente ([McGookin et al. 2010], [Sánchez García et al. 2016], [Rühmann et al. 2016] e [Forcelini et al. 2018]);

- O tamanho dos tangíveis deve ser adequado para a percepção tátil, tangíveis muito 
VIII Congresso Brasileiro de Informática na Educação (CBIE 2019)

Anais do XXX Simpósio Brasileiro de Informática na Educação (SBIE 2019)

Tabela 3. Resposta da QP1.

\begin{tabular}{|l|l|}
\hline Trabalho & Tecnologia \\
\hline Jafri (2014) & $\begin{array}{l}\text { Blocos com letras em Braille gravadas nas laterais e etiquetas NFC } \\
\text { (do inglês Near Field Communication) fixadas nas suas bases que } \\
\text { são reconhecidas por tecnologia RFID (do inglês Radio-Frequency } \\
\text { IDentification) }\end{array}$ \\
\hline $\begin{array}{l}\text { Sánchez García } \\
\text { et al. (2016) }\end{array}$ & $\begin{array}{l}\text { Blocos de montar LEGOß } \text { adaptados para o alfabeto Braille ou celas } \\
\text { Braille em EVA cujas imagens são capturadas e processadas por } \\
\text { Visão Computacional com a biblioteca OPENCV }\end{array}$ \\
\hline $\begin{array}{l}\text { Forcelini et al. } \\
(2018)\end{array}$ & $\begin{array}{l}\text { Celas Braille em EVA cujas imagens são capturadas e processadas } \\
\text { por Visão Computacional com a biblioteca OPENCV }\end{array}$ \\
\hline $\begin{array}{l}\text { Lozano et al. } \\
(2018)\end{array}$ & $\begin{array}{l}\text { Objetos tangíveis com etiquetas NFC para reconhecimento por } \\
\text { RFID }\end{array}$ \\
\hline $\begin{array}{l}\text { Maher et al. } \\
(2018)\end{array}$ & Cela Braille eletrônica que utiliza microcontroladores \\
\hline
\end{tabular}

pequenos ou ampliados podem não ser reconhecidos [Ávila-Soto et al. 2017];

- A base ou área de trabalho deve ter tamanho que permita construções significativas conforme contexto de uso [Forcelini et al. 2018];

- Usar linhas de referência na base de montagem da TUI para facilitar o alinhamento dos tangíveis [Ávila-Soto et al. 2017];

- Utilizar uma caixa organizadora para facilitar a localização dos tangíveis durante o uso da TUI [Lozano et al. 2018];

- Dar preferência às tecnologias e materiais de baixo custo para a construção dos tangíveis ([Jafri 2014], [Forcelini et al. 2018] e [Maher et al. 2018]);

- Possibilitar ao professor formas e métodos "Do-It-Yourself” para ele construir os objetos tangíveis ([Jafri et al. 2017] e [Brulé et al. 2016]);

- O design e o desenvolvimento do ambiente TUI devem ser realizados em conjunto com designers e professores de crianças cegas [Sánchez García et al. 2016];

- Prezar pela qualidade estética visual, auditiva e tátil, pois é benéfico para a inclusão e pode desencadear emoções positivas [Brulé et al. 2016];

- O processo de interação deve utilizar recursos multimodais e recursos multimídia para melhorar e enriquecer a comunicação durante o processo de utilização[Sánchez García et al. 2016];

- A TUI deve fornecer interação mais natural possível e levar em consideração as habilidades motora e cognitiva de crianças cegas[Sánchez García et al. 2016];

- O sistema deve fornecer feedback para orientar, motivar e dar autonomia para a criança durante o uso da TUI [Lozano et al. 2018];

- As atividades de letramento devem ser planejadas para serem significativas e suportar trabalhos interativos e colaboração[Sánchez García et al. 2016];

- Os cenários de uso devem ser lúdicos e envolver as crianças, de preferência via jogos ([Brulé et al. 2016] e [Forcelini et al. 2018]).

A arquitetura proposta por [Forcelini et al. 2018] e o framework proposto por [Maher et al. 2018] também podem ser usados como referência em projetos de TUIs para crianças cegas. 
VIII Congresso Brasileiro de Informática na Educação (CBIE 2019)

Anais do XXX Simpósio Brasileiro de Informática na Educação (SBIE 2019)

\subsection{Discussão}

Os resultados do MS mostraram que as TUI vem sendo utilizadas para apoiar atividades de alfabetização e letramento Braille. Entre os trabalhos do grupo 1, 2 deles são de autoria de pesquisadores brasileiros e são voltados ao apoio do letramento Braille. Os outros trabalhos são de pesquisadores estrangeiros sendo 1 deles voltado ao letramento e os demais à alfabetização. Os 3 trabalhos sobre letramento apoiam atividades de construção de textos em Braille, mas apresentam limitações para apoiar atividades de construções em Braille mais complexas como os mapas conceituais, o que demostra a relevância de atuar nesse nicho de pesquisa. Em relação as tecnologias utilizadas por esses trabalhos, os 2 trabalhos brasileiros usam a visão computacional e a biblioteca OPENCV. Já os demais trabalhos usam RFID e microcontroladores (3 trabalhos). Entre as tecnologias usadas, a visão computacional parece ser a que viabiliza uma TUI com custo mais baixo. Isso porque as webcams são mais baratas que sensores, leitores RFID e microcontroladores. Além disso, são encontradas com mais facilidade em ambientes educacionais.

Em relação aos tipos de interações que as crianças podem ter com os ambientes foi observado que é unânime o uso do toque entre os trabalhos do grupo 1. Em ambiente interativo tangível para o ensino do Braille o toque é indispensável porque o Braille é um sistema de leitura e escrita tátil. Apenas um trabalho do grupo fornece interação por meio da fala para o usuário fornecer resposta falada. Também foi observado que o feedback auditivo é unanimidade entre os trabalhos e é utilizado para conduzir e orientar o usuário sobre a realização das atividades nas TUI.

Ainda sobre os trabalhos do grupo 1, foi observado que 2 deles consultaram e entrevistaram professores de Braille para orientar o design das TUIs. Um deles não apresentou testes em protótipo e o outro testou o protótipo com professor cego. Em relação aos demais trabalhos do grupo, dois deles realizaram testes em protótipos com professores e crianças cegas e um deles não apresentou testes na funcionalidade de números Braille do protótipo. Foi observado que a participação dos professores durante o processo de design e construção se confirma como uma metodologia necessária pela comunidade científica. Isso foi percebido por um dos trabalhos dos brasileiros que traz esse fato como requisito para elaboração de TUIs para crianças cegas. Em relação aos testes com crianças cegas, parece ser a forma mais adequada e indicada de validar uma TUI voltada ao ensino do Braille, sempre que não for possível envolvê-las no próprio design.

Além disso, a partir dos trabalhos revisados (grupo 1 e grupos 2) foi possível extrair um conjunto de recomendações e requisitos que pode ser utilizado como guia para a construção de um ambiente computacional com TUI para apoiar atividades de letramento Braille.

\section{Conclusão}

Foi apresentado um MS que teve como objetivo investigar ambientes computacionais baseados em TUI para apoiar o ensino do Braille. Foram investigados trabalhos publicados nos últimos 10 anos em um conjunto de bases digitais conhecidas e consolidadas no meio científico. Dez trabalhos foram selecionados utilizando metodologia baseada em Kitchenham e Charters (2007). Essa pequena quantidade de trabalhos sugere que pouco se pesquisa e se produz em termos de TUI para o ensino do Braille. Isso, juntamente com os resultados atuais de pesquisas na área do ensino da leitura em Braille que garantem a sua 
VIII Congresso Brasileiro de Informática na Educação (CBIE 2019)

Anais do XXX Simpósio Brasileiro de Informática na Educação (SBIE 2019)

necessidade para o acesso à plena cidadania pelas pessoas cegas ([Stanfa e Johnson 2017], [Davanzo Batista 2018], [Martinez et al. 2018] e [Silverman e Bell 2018]), mostra a relevância da continuidade da pesquisa.

Os resultados deste MS mostram que as TUIs apresentadas possuem limitações em relação ao apoio de atividades que envolvem construções em Braille mais complexas como os mapas conceituais, sugerindo uma oportunidade de pesquisa a ser explorada. Como sugestão de trabalhos futuros, pode-se expandir este MS para o público cegos adultos, investigar se existem trabalhos que apresentam orientações práticas, ferramentas, ou softwares para apoiar a construção de mapas conceituais em Braille.

\section{Referências}

American Foundation for the Blind (2019). O que é Braille? https: / /www . afb. org/blindness-and-low-vision/braille/what-braille. Acessado em: 2019-05-16.

Ávila-Soto, M., Valderrama-Bahamóndez, E., e Schmidt, A. (2017). TanMath: A Tangible Math Application to support children with visual impairment to learn basic Arithmetic. In Proceedings of the 10th International Conference on PErvasive Technologies Related to Assistive Environments - PETRA '17, pages 244-245, New York, New York, USA. ACM Press.

Basili, V. e Rombach, H. (1988). The TAME project: towards improvement-oriented software environments. IEEE Transactions on Software Engineering, 14(6):758-773.

Brulé, E., Bailly, G., Brock, A., Valentin, F., Denis, G., Jouffrais, C., e Brule, E. (2016). MapSense: Multi-Sensory Interactive Maps for Children Living with Visual Impairments. In Proceedings of the Annual ACM Conference on Human Factors in Computing Systems - CHI, San José. ACM.

Davanzo Batista, R. (2018). O PROCESSO DE ALFABETIZAÇÃO DE ALUNOS CEGOS E O MOVIMENTO DA DESBRAILIZAÇÃO. PhD thesis, Universidade Metodista de Piracicaba (UNIMEP).

Forcelini, P. G., García, L. S., e Schultz, E. P. B. (2018). Braille technology beyond the financial barriers: A braille literacy platform to efectively combat braille literacy crisis. In Proceedings of the 8th International Conference on Software Development and Technologies for Enhancing Accessibility and Fighting Info-exclusion - DSAI 2018, pages 41-46, New York, New York, USA. ACM Press.

Foucambert, J. (2008). Modos de ser leitor: Aprendizagem e ensino da leitura no ensino fundamental. Editora UFPR, Curitiba.

Freire, P. (1987). A importância do ato de ler. Cortez Editora, São Paulo, 18 edition.

Ishii, H. (2008). The tangible user interface and its evolution. Communications of the ACM, 51(6):32.

Jafri, R. (2014). Electronic Braille Blocks: A Tangible Interface-Based Application for Teaching Braille Letter Recognition to Very Young Blind Children. pages 551-558. Springer, Cham.

Jafri, R., Aljuhani, A. M., e Ali, S. A. (2017). A tangible user interface-based application utilizing 3D-printed manipulatives for teaching tactual shape perception and spatial 
VIII Congresso Brasileiro de Informática na Educação (CBIE 2019)

Anais do XXX Simpósio Brasileiro de Informática na Educação (SBIE 2019)

awareness sub-concepts to visually impaired children. International Journal of ChildComputer Interaction, 11:3-11.

Kitchenham, B. e Charters, S. (2007). Guidelines for performing Systematic Literature Reviews in Software Engineering. Technical report, EBSE Technical Report EBSE2007-01, Software Engineering Group Department of Computer Science Keele University.

Lozano, M.-D., Penichet, V. M. R., Leporini, B., e Fernando, A. (2018). Tangible User Interfaces to Ease the Learning Process for Visually-Impaired Children. In Proceedings of the 32nd International BCS Human Computer Interaction Conference (HCI 2018), pages $1-5$, Belfast, UK.

Maher, R. R., Baqai, A., e Jan, M. (2018). Cost Effective Frame Work for implementing Elementary Education for Visually Impaired. In 2018 5th International Multi-Topic ICT Conference (IMTIC), pages 1-7. IEEE.

Martinez, A. B. C., Barros, A. S. S. e., e Santos, A. C. (2018). Letramentos de jovens cegos: estudo de caso em escolas do distrito de Braga. Colóquio Luso-Brasileiro de Educação - COLBEDUCA, 3(0).

McGookin, D., Robertson, E., e Brewster, S. (2010). Clutching at straws: using tangible interaction to provide non-visual access to graphs. In Proceedings of the 28th international conference on Human factors in computing systems - CHI'10, page 1715, New York, New York, USA. ACM Press.

Moreira, E. e Baranauskas, M. C. (2015). Tecnologias tangíveis e vestíveis como recursos para ambiente inclusivo: uma revisão sistemática. In Brazilian Symposium on Computers in Education (Simpósio Brasileiro de Informática na Educação - SBIE), volume 26, page 842.

Nações Unidas do Brasil (2019). Primeiro Dia Mundial do Braille destaca importância da linguagem escrita para os direitos humanos. https: // nacoesunidas.org/ primeiro-dia-mundial-do-braille-destaca-importancia-da-l $\backslash$ linguagem-escrita-para-os-direitos-humanos/. Acessado em: 2019-03-29.

Rühmann, L. M., Otero, N., e Oakley, I. (2016). A Tangible Tool for Visual Impaired Users to Learn Geometry. In Proceedings of the TEI '16: Tenth International Conference on Tangible, Embedded, and Embodied Interaction - TEI '16, pages 577-583, New York, New York, USA. ACM Press.

Sánchez García, L., Hilton Sayeg de Siqueira, J., Bueno, J., e Galera Forcelini, P. (2016). A Tangible Interaction Platform as Concrete Support for Blind Children Literacy in Braille. pages 135-146. Springer, Cham.

Silverman, A. M. e Bell, E. C. (2018). The Association between Braille Reading History and Well-being for Blind Adults. Journal of Blindness Innovation and Research, 8(1).

Soares, M. (1999). Letramento: um tema em três gêneros. Autêntica, São Paulo.

Stanfa, K. e Johnson, N. (2017). Improving Reading Fluency in Braille Readers Using Repeated Readings. Journal of Blindness Innovation and Research, 7(1). 